\title{
Determinación de parámetros óptimos de tostado de semilla de copoazú (Theobroma grandiflorum (Willd ex Spreng) Schum) para la obtención de licor o pasta
}

\section{Determination of optimal roasting parameters of copoazu seed (Theobroma grandiflorum (Willd ex Spreng) Schum) for obtaining liquor or pasta}

\author{
Cajo-Pinche, María [D 0000-0003-4753-4029]1; Díaz-Viteri, Javier [(D) 0000-0002-5384-5539]1 \\ 'Universidad Nacional Amazónica de Madre de Dios, Puerto Maldonado, Perú \\ $\triangle$ mcajo@unamad.edu.pe
}

Recibido: 06/I I/202I;

Aceptado: 06/12/2021;

Publicado: 20/01/2022

Resumen: El copoazú es uno de los cultivos de mayor importancia en Madre de Dios, departamento con mayor producción; la semilla de copoazú sirve de materia prima para chocolate de copoazú. Siendo una operación importante el tostado; etapa en la cual se desarrollan aroma, color y sabor del chocolate. Por ello es importante definir la temperatura y el tiempo óptimo de tostado de la semilla de copoazú. Se utilizó semillas con índices de fermentación bien fermentados $80 \%$ estas fueron sometidas a proceso de tostado utilizando tres temperaturas de $110^{\circ} \mathrm{C}, 120^{\circ} \mathrm{C}$ y $130^{\circ} \mathrm{C}$ por tiempos de tostado de $20 \mathrm{~min}, 30$ min y 40 min, aplicando un diseño experimental completamente al azar (DCA) con arreglo factorial $3 \times 3$, siendo los factores: la temperatura de tostado y los niveles tiempos de exposición al tostado, la pasta de copoazú fue evaluada por un panel entrenado de APPCACAO. Se evaluó los atributos de olor, acidez, amargor, astringencia, sabor y aroma, limpieza, postgusto, obteniendo puntuaciones de los atributos desde $4 \mathrm{I}$ a 60 ; bajo el tratamiento de $130^{\circ} \mathrm{C} \times 20 \mathrm{~min}$ ocurre una significancia de $95 \%$. El mejor tratamiento de tostado es de $130^{\circ} \mathrm{C} \times 20$ min ya que así se obtiene un puntaje de 60 sobre 100 respecto a los atributos del licor de copoazú; asimismo, este licor de copoazú tiene las siguientes características proximales: humedad: 1.66, proteína: 9.33 , grasa: 56.40 , ceniza: I.45, fibra: 2.72 , carbohidratos: 2916 y cadmio: 0.009

Palabras clave: características; físicas; sensoriales; temperatura, tiempo

Abstract: The copoazú in Madre de Dios is one of the most important crops, we have to note that in Peru it is the department with the highest production; copoazú seed serves as raw material for copoazú chocolate. Roasting being an important operation; stage in which the aroma, color and flavor of the chocolate develop. For this reason, it is important to define the optimum temperature and time for toasting the copoazú seed. Seeds with $80 \%$ well fermented fermentation indices were used, these were subjected to a roasting process using three temperatures of $110^{\circ} \mathrm{C}, 120^{\circ} \mathrm{C}$ and $130^{\circ} \mathrm{C}$ for roasting times of $20 \mathrm{~min}, 30 \mathrm{~min}$ and $40 \mathrm{~min}$ By applying a completely randomized experimental design (DCA) with a 3 x 3 factorial arrangement, the factors being: the roasting temperature and the levels of exposure to roasting, the copoazu paste was evaluated by a trained APPCACAO panel. The attributes of smell, acidity, bitterness, astringency, flavor and aroma, cleanliness, aftertaste were evaluated, obtaining scores of the attributes from $4 \mathrm{I}$ to 60 ; under the treatment of $130^{\circ} \mathrm{C} \times 20 \mathrm{~min}$, a significance of $95 \%$ occurs. The best roasting treatment is $130^{\circ} \mathrm{C} \times 20$ min since this gives a score of 60 out of 100 regarding the attributes of the copoazu liquor; Likewise, this copoazu liquor has the following proximal characteristics: humidity: 1.66, protein: 9.33 , fat: 56.40 , ash: I.45, fiber: 2.72 , carbohydrates: 2916 and cadmium: 0.009

Keywords: characteristics; physical; sensory; temperature; time

Cómo citar / Citation: Cajo-Pinche, M. \& Diaz-Viteri, J. (2022). Determinación de parámetros óptimos de tostado de semilla de copoazú (Theobroma grandiflorum (Willd ex Spreng) Schum) para la obtención de licor o pasta. Revista agrotecnológica amazónica, 2(I), e265. https://doi.org/I0.5I252/raa.v2il.265 


\section{Introducción}

El copoazú (Theobroma grandiflorum (Willd ex Spreng) Schum), conocido como cacao amazónico; "es un árbol típicamente amazónico que produce frutos carnosos, pertenece a la familia de las Malvaceae, que se encuentra en estado silvestre en la parte sur y sur-este de la amazonia oriental" (Giorgini Augusto Venturieri \& Aguiar, 1988)(Aldave Palacios, 2016)(G.A. Venturieri, 1990), planta típica de la región Madre de Dios en Perú. Después de todo, el Theobroma Grandiflorum ya lleva algunos años siendo anunciado como la próxima "superfruta", debido a sus numerosos nutrientes y antioxidantes (Wallace, 2012). La Asociación de Agricultores Agropecuarios de la Comunidad El Progreso de Madre de Dios, organización que realizó su primera exportación de 15 toneladas de semilla seca de copoazú a Rusia (Agencia Agraria de Noticias, 2020) a través de la Cooperativa Sur Oriente. Constituyendo un agente económico en la región Madre de Dios.

Según Dirección Regional de Agricultura (202I) el copoazú tiene un rendimiento I 352 $\mathrm{Kg} / \mathrm{Ha}$, en el 2020 fue de 36 ha y este 202I hasta julio ya van 4I ha sembradas esto implica que será mayor la producción de copoazú en Madre de Dios. Asimismo, El Gobierno Regional de Madre de Dios promueve la producción del copoazú un fruto oriundo del Brasil, cuyo aroma y sabor es similar al del cacao (Agencia Agraria de Noticias, 2020). Cáritas Madre de Dios, el copoazú ha sido catalogado como uno de los frutos más promisorios de la Amazonía tiene gran aceptación en el mercado regional, donde se consume en forma de refrescos, helados, yogur, chupetines y en tragos exóticos, elaborados a partir de su pulpa (Agencia Agraria de Noticias, 2020) (Quintero, 2020).

Del fruto, similar al coco en apariencia, también se puede aprovechar su almendra para elaborar chocolate, como se hace en Brasil y Bolivia. Tiene muy buenos contenidos de grasas e incluso mejores que el cacao, de acuerdo con información nutricional brindada por Caritas Madre de Dios, el copoazú posee calcio, fósforo y vitamina $C$, entre otros compuestos. rendimiento puede alcanzar de $1500 \mathrm{~kg}$ de pulpa por Ha y su precio, $\mathrm{S} / .8 \mathrm{Kg}$ en temporada, es decir, entre enero y marzo. Por año suelen obtener entre 50 y $60 \mathrm{mil} \mathrm{Kg}$, La composición porcentual del fruto fresco de copoazú es: Cáscara 46.47\%, Pulpa $36.79 \%$, Almendras $16.74 \%$ (Giorgini Augusto Venturieri \& Aguiar, 1988).

El tostado es; "una operación que contribuye a disminuir la humedad de las semillas por debajo del $2 \%$, en el caso del cacao estas condiciones facilitan su desprendimiento y las semillas son más rígidas facilitando su posterior molienda, además durante el tostado se producen reacciones de Maillard que desarrollan características finales de color, sabor y aroma a chocolate, es decir definir sabores del cacao (Domínguez-Pérez et al., 2019). Un buen tostado facilita la eliminación de microorganismos y ácidos volátiles que generen sabores amargos y ácidos. Para ello se exponen las semillas a temperaturas de entre $110^{\circ} \mathrm{C}$ a $150^{\circ} \mathrm{C}$ por tiempos de entre 15 min a 60 min" (Alegría Vargas, 20I5).

En la producción de licor o pasta de copoazú materia prima del chocolate, es importante definir la temperatura y el tiempo óptimo de tostado de la semilla de copoazú ya que estos parámetros nos permitirán buen aroma, color y sabor. En Madre de Dios se produce de forma tradicional ya que los parámetros de tostado relacionados a la temperatura y tiempo no son medidos y controlados repercutiendo en sus características organolépticas y fisicoquímicas, por lo que su calidad hace difícil que sea introducido en el mercado nacional e internacional. El interés 
de optimizar los parámetros del tostado, es para obtener un chocolate de calidad como lo exige el mercado en la actualidad, de esta manera contribuir con el aporte tecnológico a los empresarios que se dedican a este rubro para que puedan incrementar sus ventas, incursionar en otros mercados, etc.

La semilla de copoazú, tiene un alto potencial para la obtención de chocolate y manteca de copoazú. A la fecha no se ha desarrollado investigaciones que permitan estandarizar los productos antes mencionados, que en pocas cantidades se procesan. Es por ello que la presente investigación pretendio determinar: parámetros óptimos de temperaturas y tiempos de exposición del tostado de la semilla, operación muy importante que permite obtener un chocolate con buenas características físicos químicos y organolépticos y de alto valor nutritivo. Generando así un alto valor agregado y un potencial agroindustrial. Generando mayores ingresos a los empresarios dedicados a este rubro ya que podrán entrar en el mercado nacional e internacional; siendo el principal beneficiario el consumidor final.

Durante la investigación se tuvo limitantes en cuanto a la poca información respecto a los antecedentes de trabajos realizados en cuanto al: tostado de la semilla de copoazú y la estacionalidad de la fruta.

El objetivo principal fue Determinar los parámetros óptimos de tostado de la semilla de copoazú (Theobroma grandiflorum (Willd ex Spreng) Schum), para la obtención de un licor o pasta de copoazú de calidad con buenas características organolépticas, propiedades físico químicas y con alto valor nutritivo.

\section{Materiales y métodos}

Las semillas de copoazú provenientes del fundo Yanahoca, Asociación de Agricultores Agropecuarios "El Progreso", ubicado en Unión Progreso, km 85 carretera Puerto Maldonado - Cusco, distrito de Inambari, provincia de Tambopata, departamento de Madre de Dios, que está ubicado a $238 \mathrm{~m} \mathrm{~s}, \mathrm{n}$. m. de altitud, latitud Sur: $12^{\circ} 52^{\prime} 48^{\prime \prime} \mathrm{S}$ y longitud Oeste: $69^{\circ} 49^{\prime} 35 . \mathrm{I}^{\prime \prime}$ W. El presente proyecto se realizó en la Planta Piloto de Frutas Tropicales de la Universidad Nacional Amazónica de Madre de Dios. El estudio fue de tipo aplicativo, de corte experimental con un diseño experimental completamente al azar (DCA) con arreglo factorial $3 \times 3$, siendo los factores: la temperatura de tostado $\left(110^{\circ} \mathrm{C}, 120^{\circ} \mathrm{C}\right.$ y $\left.130{ }^{\circ} \mathrm{C}\right)$ y los niveles tiempos de exposición al tostado ( $20 \mathrm{~min}, 30 \mathrm{~min}$ y $40 \mathrm{~min}$ ), cada tratamiento se efectuó por triplicado. se utilizó el programa estadístico design-expert (stat ease. uk), el universo fue de 100 kilos frutos de copoazú con una muestra de $20 \mathrm{~kg}$, para caracterizar la semilla de copoazú se procedió a pesar, medir el volumen y la densidad en una probeta con agua; asimismo, se tomó medidas de longitud y espesor. Una vez caracterizadas las semillas de copoazú se procedió a la obtención del licor de copoazú. Luego las muestras de licor de copoazú fueron envasados en bolsas laminadas y enviados a APPCACAO para su evaluación sensorial, ya que en Perú no existe catadores especializados en copoazú y siendo el cacao un Theobroma se optó por ello. Se debe seguir un esquema ordenado: diseño, ámbito, periodo de estudio, población y muestra, fuentes de información, intervenciones (caso de estudios experimentales), instrumentos, variables (dependientes e independientes), aspectos éticos y plan estadístico. 
Figura I. Flujograma de obtención de chocolate de copoazú

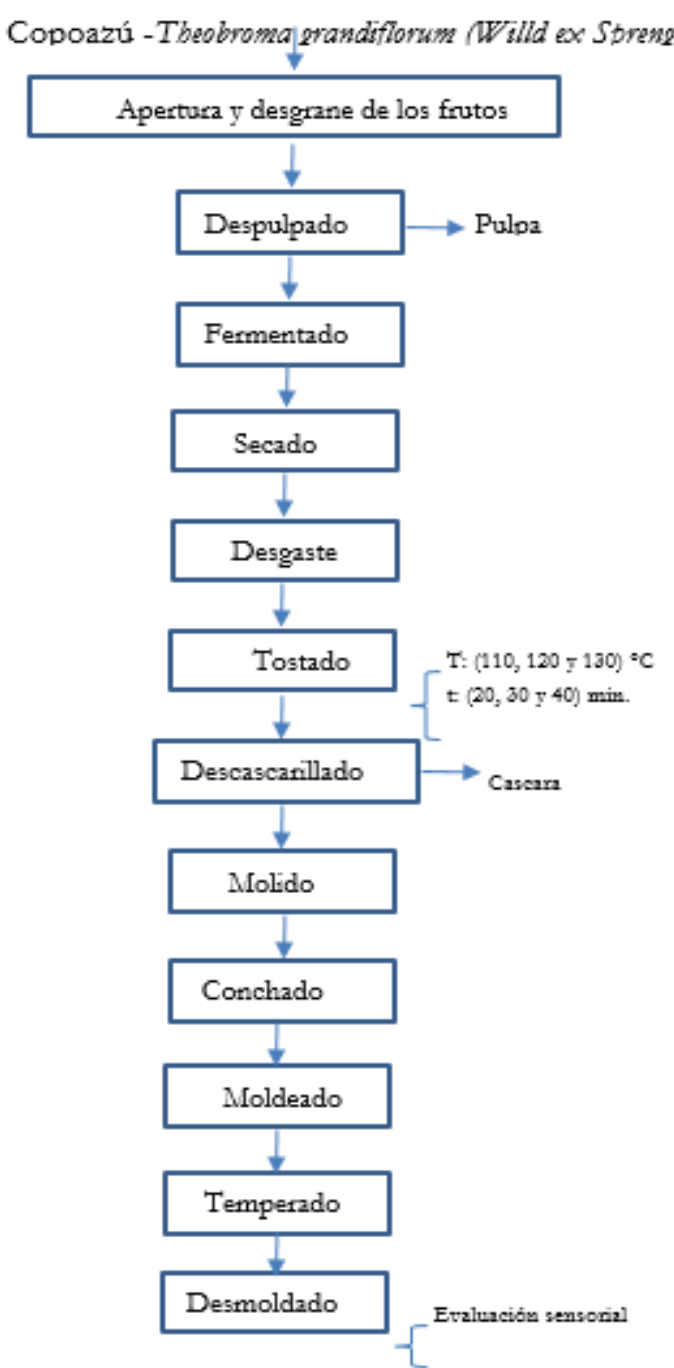

\section{Descripción del flujograma}

Material: El material vegetal de copoazú se adquirió del fundo del Yanahoca, Unión Progreso, km 85 carretera Puerto Maldonado - Cusco, distrito de Inambari, provincia de Tambopata, departamento de Madre de Dios.

Materia prima: debe ser homogénea en cuanto al tamaño, grado de madurez y variedad, excepto de daños físicos.

Apertura y desgrane de los frutos: Se realizó de forma manual, golpeando el fruto con un mazo de madera, para facilitar la apertura de este; luego se retiró las semillas de la cascara.

Despulpado: fue mecánico, para ello se utilizó la despulpadora, separando la pulpa de la semilla; la pulpa fue de $70 \%$ y lo restante semilla. Y la semilla se destinó a la fermentación.

Fermentado: Se realizó en cajones de madera rectangulares de $40 \mathrm{~cm} \times 40 \mathrm{~cm}$. y $50 \mathrm{~cm}$. de profundidad, los cajones contaron con aberturas en la parte interna y media que facilitaron el drenado del licor; asimismo se hizo la toma de temperatura y $\mathrm{pH}$ en un punto medio por 
triplicado, a partir del día cero hasta el día siete. La masa utilizada fue de $40 \mathrm{~kg}$. de almendra húmeda por cada tratamiento. Con remoción cada 24 horas

Secado: Los granos fermentados se secaron a temperatura ambiente por aproximadamente 4 días en día soleado y en días nublados siete días, en mesa de acero inoxidable. La exposición solar durante 3 horas en el día y en la noche bajo ventilación.

Desgaste: se realizó en una maquina peladora centrifuga de capacidad de $500 \mathrm{~kg} / \mathrm{hora}$, con una potencia de $2 \mathrm{hp}$, velocidad de rotación del cilindro $36 \mathrm{rpm}$ eficiencia del equipo de 78 a $86 \%$ por 4 min.

Tostado: se realizó en una estufa con capacidad de 5 litros, con un rango de 5 a $250{ }^{\circ} \mathrm{C}$, de 220 voltios, a temperaturas $\left(110^{\circ} \mathrm{C}, 120^{\circ} \mathrm{C}\right.$ y $\left.130^{\circ} \mathrm{C}\right)$ y tiempos de $20 \mathrm{~min}, 30 \mathrm{~min}$ y $40 \mathrm{~min}$.

Descascarillado: se realizó de forma manual con ayuda de tijeras

Molido: se realizó en un molino artesanal.

Conchado: se realizó en APPCACAO en un canchador de piedras nivel laboratorio con capacidad de carga de $4 \mathrm{~kg}$. Por un tiempo de $18 \mathrm{~h}$ de conchado.

Moldeado: en moldes de policarbonato.

Temperado: se congelo por $10 \mathrm{~min}$ en la nevera y luego se refrigero por $10 \mathrm{~min}$. Esta operación se realiza para la dureza del chocolate y para la estabilidad de la grasa (a mayor grasa el temperado es menor).

Desmoldado: se retiró de los moldes, asimismo se realizó la evaluación sensorial con un panel entrenado.

\section{Análisis de datos}

El diseño experimental completamente al azar (DCA) con arreglo factorial $3 \times 3$, siendo los factores: la temperatura de tostado $\left(110^{\circ} \mathrm{C}, 120^{\circ} \mathrm{C}\right.$ y $\left.130^{\circ} \mathrm{C}\right)$ y los niveles tiempos de exposición al tostado ( $20 \mathrm{~min}, 30 \mathrm{~min}$ y $40 \mathrm{~min}$ ), cada tratamiento se efectuó por triplicado. Se utilizó el programa estadístico Design-Expert (Stat Ease. UK).

\section{Resultados y discusión}

\section{Características físicas de la semilla de copoazú}

Tabla I. Característica física de la semilla de copoazú (T. grandiflorum)

\begin{tabular}{|c|c|c|c|c|c|}
\hline $\begin{array}{c}\text { Largo } \\
(\mathbf{m m})\end{array}$ & $\begin{array}{c}\text { Espesor } \\
\mathbf{( m m})\end{array}$ & $\begin{array}{c}\text { Ancho } \\
(\mathbf{m m})\end{array}$ & $\begin{array}{c}\text { Peso } \\
\text { promedio } \\
\mathbf{( g )}\end{array}$ & $\begin{array}{c}\text { Volumen } \\
\left.\mathbf{( c m}^{\mathbf{3}}\right)\end{array}$ & Densidad \\
\hline 26,98 & $|I, 7|$ & 22,29 & 5,84 & 5,89 & 0,99 \\
\hline
\end{tabular}

En la Tabla I se presentan los datos promedio respecto a las características físicas de la semilla de copoazú.

Las características físicas de la semilla de copoazú usadas para la pasta o licor de copoazú son en promedio las que se muestran en la Tabla I que coinciden con los reportados por Rojas 
Corrales \& Villagra Halanocca (2016) y Zapata Ortíz et al. (1996) los cuales presentaron datos de longitud de la semilla de $22 \mathrm{~mm}$ a $35 \mathrm{~mm}$, y ancho entre $15 \mathrm{~mm}$ a $28 \mathrm{~mm}$.

\section{Evaluación sensorial del licor de copoazú T. grandiflorum.}

Tabla 2. Atributos sensoriales del licor de copoazú T. grandiflorum.

\begin{tabular}{|c|c|c|c|c|c|c|c|c|c|}
\hline & \multicolumn{3}{|c|}{$I 10^{\circ} \mathbf{C}$} & \multicolumn{3}{c|}{$120^{\circ} \mathbf{C}$} & \multicolumn{3}{c|}{$130^{\circ} \mathbf{C}$} \\
\hline Atributos & $\mathbf{2 0}$ & $\mathbf{3 0}$ & $\mathbf{4 0}$ & $\mathbf{2 0}$ & $\mathbf{3 0}$ & $\mathbf{4 0}$ & $\mathbf{2 0}$ & $\mathbf{3 0}$ & $\mathbf{4 0}$ \\
\hline Olor & 5 & 6 & 6 & 5 & 5 & 5 & 7 & 7 & 5 \\
\hline Acidez & 5 & 5 & 4 & 6 & 5 & 5 & 5 & 5 & 5 \\
\hline Amargor & 5 & 4 & 5 & 3 & 4 & 5 & 7 & 5 & 5 \\
\hline Astringencia & 2 & 3 & 3 & 4 & 5 & 7 & 7 & 7 & 4 \\
\hline Sabor y aroma & 8 & 10 & 9 & 10 & 10 & $I I$ & 12 & 12 & 8 \\
\hline Limpieza & 10 & 10 & 10 & 10 & 10 & 10 & 9 & 10 & 10 \\
\hline Postgusto & 2 & 3 & 5 & 3 & 7 & 3 & 6 & 6 & 4 \\
\hline Puntaje del catador & 4 & 6 & 5 & 4 & 6 & 6 & 7 & 7 & 4 \\
\hline Puntuación de atributos & $4 \mathrm{I}$ & 47 & 47 & 45 & 52 & 52 & 60 & 59 & 45 \\
\hline
\end{tabular}

Fuente: APPCACAO (2017)

Tabla 3. Análisis de varianza de un factor - resumen

\begin{tabular}{|c|c|c|c|c|}
\hline Grupos & Cuenta & Suma & Promedio & Varianza \\
\hline $20 / 110^{\circ} \mathrm{C}$ & 8 & $4 I$ & 5.125 & $7.55357 / 43$ \\
\hline $30 / 110^{\circ} \mathrm{C}$ & 8 & 47 & 5.875 & $7.8392857 I$ \\
\hline $40 / 110^{\circ} \mathrm{C}$ & 8 & 47 & 5.875 & $5.8392857 I$ \\
\hline $20 / 120^{\circ} \mathrm{C}$ & 8 & 45 & 5.625 & $8.267857 I 4$ \\
\hline $30 / 120^{\circ} \mathrm{C}$ & 8 & 52 & 6.5 & $5.42857 I 43$ \\
\hline $40 / 120^{\circ} \mathrm{C}$ & 8 & 52 & 6.5 & $7.42857 I 43$ \\
\hline $20 / 130^{\circ} \mathrm{C}$ & 8 & 60 & 7.5 & $4.57 I 42857$ \\
\hline $30 / 130^{\circ} \mathrm{C}$ & 8 & 59 & 7.375 & $5.982 I 4286$ \\
\hline $40 / 130^{\circ} \mathrm{C}$ & 8 & 45 & 5.625 & $4.8392857 I$ \\
\hline
\end{tabular}

Tabla 4. Análisis de varianza de un factor

\begin{tabular}{|c|c|c|c|c|c|c|}
\hline $\begin{array}{c}\text { Origen de las } \\
\text { variaciones }\end{array}$ & $\begin{array}{c}\text { Suma de } \\
\text { cuadrados }\end{array}$ & $\begin{array}{c}\text { Grados } \\
\text { de } \\
\text { libertad }\end{array}$ & $\begin{array}{c}\text { Promedio } \\
\text { de los } \\
\text { cuadrados }\end{array}$ & F & Probabilidad & $\begin{array}{c}\text { Valor } \\
\text { crítico } \\
\text { para F }\end{array}$ \\
\hline Entre grupos & 42.1944444 & 8 & 5.27430556 & 0.8219697 & 0.58627118 & 2.08918504 \\
\hline $\begin{array}{c}\text { Dentro de los } \\
\text { grupos }\end{array}$ & 404.25 & 63 & 6.41666667 & & & \\
\hline Total & 446.444444 & 71 & & & & \\
\hline
\end{tabular}

En la Tabla 2 se puede ver que hay 04 muestras aceptables con un promedio de puntaje superior a los 50 puntos, destacando el tratamiento de tostado de $130^{\circ} \mathrm{C} \times 20 \mathrm{~min}$. Es el que obtuvo mayor puntaje respecto a los atributos sensoriales seguido del tratamiento de tostado de 130 ${ }^{\circ} \mathrm{C} \times 20$ min. Este resultado comparado con el estudio realizado por Aldave Palacios (2016) en cuanto a la temperatura de $130^{\circ} \mathrm{C}$ es igual a lo obtenido en tostado de cacao (Theobroma cacao L.) CCN-5I e ICS-6, procedentes del distrito de Uchiza, provincia de Tocache, departamento de San Martín, en Perú. Diferente a lo reportado por Moncada Rodríguez \& Hernández (2006) quienes recomiendan para el tostado de cacao silvestres y copoazú (Theobroma grandiflorum), 
parámetros óptimos de 14 min por $140^{\circ} \mathrm{C}$, se podría indicar que a mayor temperatura menor tiempo. Esto podría deberse que en nuestro caso se realizó desgaste de la cascara antes del tostado.

\section{Conclusiones}

Se estudiaron tres temperaturas de $110^{\circ} \mathrm{C}, 120^{\circ} \mathrm{C}$ y $130^{\circ} \mathrm{C}$ y tres tiempos de $20 \mathrm{~min} .30 \mathrm{~min}$. y 40 min para la operación de tostado de los cuales el mejor tratamiento para el tostado de semilla de copoazú fue $130{ }^{\circ} \mathrm{C} \times 20 \mathrm{~min}$. Habiendo alcanzado el puntaje de 60 , puntaje que corresponde a la suma de las calificaciones de los atributos: olor, acidez, amargor, astringencia, sabor y aroma, limpieza, postgusto

Se determinó las características físicas promedios de la semilla de copoazú, utilizadas para la obtención de licor de copoazú; longitud de $26,98 \mathrm{~mm}$, espesor de $1 \mathrm{I}, 7 \mathrm{I} \mathrm{mm}$, ancho de 22,29 $\mathrm{mm}$, peso promedio de $5,84 \mathrm{~g}$, volumen de $5,89 \mathrm{~cm} 3$ y densidad de $0,99 \mathrm{~g} / \mathrm{cm} 3$.

\section{Agradecimiento}

A la Universidad Nacional Amazónica de Madre de Dios, por financiar esta investigación y por facilitarnos la Planta Piloto de Frutas Tropicales donde se desarrolló la parte experimental.

\section{Referencias bibliográficas}

Agencia Agraria de Noticias. (2020). Productores de Madre de Dios exportaron 15 toneladas de copoazú a Rusia. Agraria.Pe. https://agraria.pe/noticias/productores-de-madre-de-diosexportaron-15-toneladas-de-copo-21910

Aldave Palacios, G. J. (20I6). Efecto de la temperatura y tiempo de tostado en los caracteres sensoriales y en las propiedades químicas de granos de cacao (Theobroma cacao L.) procedente de Uchiza, San Martín - Perú para la obtención de NIBS [Universidad Nacional Mayor de San Marcos]. https://hdl.handle.net/20.500.12672/5009

Alegría Vargas, E. A. (20I5). Evaluación de tratamientos previos al proceso de tostado de semillas de cacao para el diseño del área de producción de pasta de cacao (Theobroma cacao) [Escuela Politécnica Nacional]. http://bibdigital.epn.edu.ec/handle/I5000/9/30

Dirección Regional de Agricultura. (2021). Información Agrícola. DRA. https://www.dramdd.gob.pe/informacion-agricola/

Domínguez-Pérez, L. A., Lagunes-Gálvez, L. M., Barajas-Fernández, J., Olán-Acosta, M. de los Á., García-Alamilla, R., \& García-Alamilla, P. (2019). Caracterización vibracional de grupos funcionales en granos de cacao durante el tostado usando espectroscopía de infrarrojo por transformada de Fourier. Acta Universitaria, 29, I-17. https://doi.org/10.15174/au.2019.2172

Moncada Rodríguez, L. M., \& Hernández, M. S. (2006). Obtención de una cobertura de chocolate a partir de cacao silvestres, copoazú (Theobroma grandiflorum), y maraco (Theobroma bicolor), de la amazonia co-lombiana. [Universidad de la Salle]. 
https://ciencia.lasalle.edu.co/ing_alimentos/I I0/

Quintero, M. L. (2020). El copoazú: Ficha técnica, propiedades,beneficio, para la piel. Del Amazonas. https://delamazonas.com/plantas/frutas/copoazu/

Rojas Corrales, A. V., \& Villagra Halanocca, J. (2016). Evaluación de los métodos de fermentación y secado para el beneficio de semilla del copoazu (theobroma grandiflorum) y sus efectos en la calidad de pasta de chocolate natural en la provincia de tambopata-m.d.d [Universidad Nacional Amazónica de Madre de Dios]. http://repositorio.unamad.edu.pe/handle/UNAMAD/2I2

Venturieri, G.A. (1990). Variabilidade em plantas jovens de cupuacu (Theobroma grandiflorum (Willdenow ex Sprengel) Schumann) estimada por descritores morfologicos fisiologicos e isoenzimaticos e sua utilizacao em caracterizacao de germoplasma. Manaus.

Venturieri, Giorgini Augusto, \& Aguiar, J. P. L. (1988). Composição do chocolate caseiro de amêndoas de cupuaçu (Theobroma grandiflorum (Willd ex Spreng) Schum). Acta Amazonica, I8(I-2), 3-8. https://doi.org//0.1590//809-4392/988/82008

Wallace, A. (2012). El copoazú quiere ser la próxima superfruta amazónica. BBC News Mundo. https://www.bbc.com/mundo/ultimas_noticias/20/2/06/I20625_colombia_copoazu_fruta _aw

Zapata Ortíz, J. A., Pereira, A. E., Varón, E., Cárdenas, C., Cadena, F. M., \& Rojas González, S. (1996). El cultivo de copoazú (Theobroma grandiflorum) en el Piedemonte Amazónico colombiano. AGROSAVIA. http://hdl.handle.net/20.500.12324/31918

\section{Financiamiento}

Ninguno.

\section{Conflicto de intereses}

La presente publicación no presenta conflicto de intereses.

\section{Contribución de autores}

Cajo-Pinche, María: Investigador y redactor del presente artículo.

Díaz-Viteri, Javier: Investigador y redactor del presente artículo. 